\title{
Servicios ambientales en los tiempos de la bloeconomía forestal. Retos y oportunidades en el monte mediterráneo
}

\author{
Ovando, P. \\ Swiss Federal Institute for Aquatic Science and Technology (eawag).
}

\begin{abstract}
Resumen
Recientemente se ha rescatado el término bioeconomía como paraguas interdisciplinar para la gestión de un desarrollo socioeconómico sostenible a través del uso eficiente de los recursos naturales. Las estrategias de desarrollo basadas en el sector forestal para una futura bioeconomía en Europa buscan satisfacer una creciente demanda de materiales y energía renovables. La transformación del sector forestal hacia estos últimos objetivos exige encontrar nuevas opciones y ventajas competitivas que mantengan la viabilidad futura del sector. El desarrollo de una nueva industria forestal sustentable en el monte mediterráneo se enfrenta a varios retos, que pueden verse magnificados en condiciones socioeconómicas y ambientales cambiantes e inciertas. Uno de estos, es la necesidad de promover formas de gestión y tecnologías que incrementen la productividad y rentabilidad de los recursos forestales y sean compatibles con la provisión, en calidad y cantidad suficientes, de servicios ambientales que fluyen de los ecosistemas forestales y son relevantes para el bienestar social. En este trabajo se examinan potenciales estrategias bioeconómicas para el sector forestal mediterráneo, a partir de la revisión de literatura especializada, con especial atención a los retos y oportunidades para la provisión de servicios ambientales clave en los montes mediterráneos, como son la regulación hídrica o la protección de la biodiversidad. Este trabajo ofrece algunas recomendaciones para compatibilizar la producción de bienes y servicios comerciales y ambientales en montes, a la vez que identifica líneas de investigación futuras para mejorar nuestro conocimiento sobre los potenciales efectos ambientales, sociales y económicos de nuevas formas de gestión bioeconómica de los montes mediterráneos.
\end{abstract}

Palabras clave: Servicios ambientales, bioenergía, biomasa, nuevos materiales, impactos ambientales y económicos. 


\section{Introducción}

El término bioeconomía fue acuñado por el economista Nicholas Georgescu-Roegen en los años 60 del siglo pasado, con la idea de dotar al pensamiento económico de un nuevo estilo dialéctico, y una nueva disciplina capaz de combinar elementos de la economía convencional con la biología y la termodinámica; atendiendo al origen biológico y a las limitaciones físicas del desarrollo económico (Mayumi, 2001). Este término ha sido rescatado recientemente como paraguas interdisciplinar para la gestión de un desarrollo socioeconómico sostenible a través del uso eficiente de los recursos naturales. De forma más concreta, este término define un nuevo modelo de desarrollo basado en la producción sustentable de recursos renovables -terrestres, pesqueros y acuáticos- para su conversión en alimentos, forrajes, fibras vegetales, bioenergía, y servicios públicos (Hetemäki, 2014).

La bioeconomía como modelo de desarrollo es una respuesta de la industria y las instituciones a las actuales crisis ambiental y social, al cambio climático y al temor a la futura escasez de combustibles fósiles (al menos a precios asequibles); que están forzando cambios sustanciales en las estrategias empresariales, y regionales de desarrollo. Estas nuevas estrategias básicamente pretenden encontrar y fomentar alternativas viables a los combustibles y materias primas no renovables, sobre la base del conocimiento y desarrollo tecnológico. En principio, la implantación de un modelo bioeconómico entrañaría modificaciones significativas en las formas de producción, pero también de consumo, a fin de reducir la alta dependencia de recursos no renovables y las emisiones de gases de efecto invernadero que caracterizan el modelo económico actual.

La transformación productiva hacia un modelo bioeconómico no es ajena al sector forestal europeo, y este cambio de rumbo podría conllevar oportunidades relevantes para la dinamización y diversificación de un sector que ha venido experimentando dificultades en las últimas décadas (FTP, 2013). El sector forestal en Europa ha ido perdiendo gradualmente su importancia relativa en los mercados internacionales de productos forestales (e.g. pulpa y papel o madera de sierra) desde el año 2000, a la vez que países emergentes, como China, Brasil, Indonesia o Rusia, han incrementado su peso en los mercados globales de estos productos. La crisis financiera de 2008 ha agudizado esta tendencia, conllevando pérdidas de empleo mayores a las experimentadas por el sector manufacturero en Europa (Hetemäki y Hurmekoski, 2014). La respuesta del sector forestal europeo a esta situación es todavía tímida, pero se están gestando algunos cambios en el mismo, tendientes a su diversificación e interconexión con otros sectores económicos, como la construcción o las industrias química, energética o textil. En principio, se espera que estos cambios conduzcan a la transformación de los modelos de producción y mercados forestales en los próximos 20 a 30 años (Duchesne y Wetzel, 2003; Hetemäki et al., 2014).

Las oportunidades más prometedoras para la transformación productiva del sector forestal europeo están, hoy en día, vinculadas a las estrategias climática ${ }^{1}$ y ener-

${ }^{1}$ El principal objetivo de la política climática, para 2030, es la reducción de emisiones de gases de efecto invernadero en un $40 \%$ respecto de las emisiones de 1990, en la Unión Europea (UE). 
gética europea (Comisión Europea, 2014). De hecho la UE espera que el uso de biomasa para la producción de energía crezca en las próximas décadas para cumplir los objetivos vinculantes que suponen las cuotas del $20 \%$ y $27 \%$ de energía procedente de fuentes renovables en el consumo total de energía en 2020 y 2030, respectivamente. La consecución de estas ambiciosas metas requiere, en principio, profundizar y continuar los cambios en la oferta y consumo energético (Blennow et al., 2014). Y la producción y procesamiento de productos leñosos es, potencialmente, uno de los elementos clave para cumplir los anteriores objetivos políticos (Ovando y Caparrós, 2009; Kellomäki et al. 2013; Sikkema et al., 2016). De hecho, la biomasa sólida para la producción de calor (calefacción y agua caliente) y electricidad es la principal fuente de energía primaria renovable en la UE (Solid Biomass Barometer, 2015; Eurostat, 2017), representando hoy en día casi la mitad del consumo de energías renovables en esta región (Comisión Europea, 2013).

La industria forestal, especialmente en países del norte de Europa, está iniciando cambios en los modelos de negocio, a través de la integración de productos como biocombustibles (Huang et al. 2010; Olesen et al., 2016), nuevos materiales de construcción basados en productos leñosos (Häyrinen et al. 2017), o telas, plásticos y productos químicos basados en celulosa, lignina, resinas o ceras de origen forestal (Babu et al. 2013; Sjostrom, 2013; Scarlat et al. 2015) entre otros. Estas nuevas demandas podrían suponer un incremento en la movilización de biomasa y en la intensidad de manejo de los bosques europeos (Framstad et al., 2009) o la conversión de hábitats de alto valor ambiental en tierras productivas (Pedroli et al., 2013); cuyas políticas se han orientado, en las últimas dos décadas, más bien a favorecer su conservación y multifuncionalidad. Entendido este último concepto como la búsqueda de un equilibrio entre las funciones sociales, económicas y ambientales de los ecosistemas forestales (Comisión Europea, 2010). Y considerando además que los ecosistemas forestales desempeñan un papel esencial en la mitigación del cambio climático, la conservación de la biodiversidad, el suministro de agua dulce, productos forestales no madereros y la provisión de entornos recreativos (Comisión Europea, 2013).

Las tasas de extracción maderera en los bosques europeos no superan el 75\% de su crecimiento anual (Nabuurs et al. 2007; MCPFE et al. 2007), por lo que todavía existe margen para incrementar la extracción sostenible de madera. No obstante, uno de los principales retos a los que se enfrenta la nueva estrategia forestal bioeconómica es cómo compatibilizar la previsible intensificación en la gestión forestal y una mayor movilización de madera con el mantenimiento de la multifuncionalidad de los bosques. Esta pregunta es especialmente relevante en cuanto a la provisión de servicios ambientales públicos, como la conservación de la biodiversidad (Söderberg y Eckerberg, 2013), la provisión de opciones de uso recreativo o valores paisajísticos (Framstad et al., 2009; Comisión Europea, 2010), la regulación de la calidad hídrica (Eriksson et al. 2011), y otros servicios ambientales publicos fuera del mercado asociados a la proteccion de suelos, control de la erosion, avalanchas o deslizamientos de tierra (European Commission, 2012).

El sector forestal europeo es diverso, y pueden observarse claras diferencias en las características ecológicas, productivas, económicas e institucionales de los bos- 
ques del centro, norte, noroeste, sur oeste, este y sur este de Europa (MCPFE et al. 2007). Una parte relevante de la incipiente literatura que analiza el potencial, las oportunidades y retos para el desarrollo de una industria forestal bioeconómica está claramente dominada por estudios de caso y expertos del norte y noroeste de Europa (Häyrinen et al., 2013; Hetemamäki, 2014; Sikkema et al., 2016); incluyendo países como Suecia, Finlandia, Francia o Alemania, con mayor potencial para la producción forestal tanto por su superficie boscosa, como por su productividad (Ericsson y Nilsson, 2006; MCPFE et al. 2007; Mola-Yudego et al., 2017). Todavía son escasos los estudios que analizan la adopción de nuevos modelos de producción bioeconómicos en los montes mediterráneos de Europa, y estos se focalizan fundamentalmente en el análisis de su potencial bioenergético (e.g. López-Rodríguez et al., 2009; Rodríguez-Pleguezuelo et al., 2014; Manolis et al., 2016; Paredes-Sánchez, et al. 2016; Turrado et al. 2016).

En principio, las oportunidades y, especialmente, los retos para la implantación de un modelo forestal bioenergético en la Europa mediterránea son diferentes a los esperados en bosques del centro y norte de Europa, que, en principio requerirían de estrategias diferenciadas. Un primer elemento a destacar son las condiciones climáticas particulares de la región, que la hacen más vulnerable a situaciones climáticas extremas y cambiantes, que pueden afectar el stress y déficit hídricos (Schroter, 2005; García-Ruiz et al., 2011), el crecimiento del arbolado (Vicente-Serrano et al. 2010) e incrementar el riesgo de incendios forestales (Palahi et al. 2008). Un segundo elemento está ligado a la multiplicidad de usos de los montes mediterráneos que integran simultáneamente actividades agrícolas, ganaderas, forestales, cinegéticas, normalmente extensivas, y con la producción de servicios con una demanda creciente, como son los usos recreativos o la conservación de especies y hábitats (e.g. Campos et al., 2008a; Ovando et al. 2016). Estos usos agroforestales han sido, y todavía son, elementos de modelización y conservación del paisaje mediterráneo (San Miguel-Ayanz, 2005), por lo que una intensificación o una simplificación de los sistemas de producción podría afectar su equilibrio y las funciones ecológicas, sociales y económicas del monte mediterráneo, incluida la provisión de servicios ambientales.

\section{Objetivos}

Este artículo ofrece una reflexión crítica sobre la implantación estrategias de desarrollo bioeconómico en el monte mediterráneo, con especial atención a los eventuales conflictos entre una potencial intensificación productiva y la provisión de servicios ambientales públicos. Este trabajo discute dos preguntas clave para el desarrollo de una nueva bioeconomía forestal en la región mediterránea:

- ¿Cuáles son los principales retos y oportunidades para el desarrollo de una bioeconomía forestal competitiva y sustentable en el monte mediterráneo?

- ¿Cuál es el papel de los servicios ambientales en una bioeconomía forestal mediterránea? 


\section{Hacia una nueva economía forestal}

\subsection{Estrategia bioeconómica europea para el sector forestal}

La estrategia bioeconomía de la Unión Europea fue publicada en febrero de 2012, con el propósito de dirigir los esfuerzos comunitarios hacia la diversificación y una rápida adaptación económica a los retos globales y regionales futuros. Esta estrategia busca promover la producción de recursos biológicos renovables y su conversión en productos vitales (i.e. alimentos, forrajes, fibras alimentarias) y bioenergía. Entre sus objetivos, están el reconciliar la seguridad alimentaria en la UE con en el uso sustentable de los recursos renovables para propósitos industriales, la protección ambiental, la generación de nuevas oportunidades de empleo y competitividad, y, de forma prioritaria, la reducción de la alta dependencia (y vulnerabilidad) de la economía europea de los combustibles fósiles (European Commission, 2012).

En cuanto al sector forestal, el documento que desarrolla esta estrategia indica que se espera que la demanda de madera y biomasa para la producción energética estimule mejoras en las gestiones forestales, dirigidas a incrementar la productividad de los bosques, y a movilizar una mayor cantidad de madera y residuos forestales. Asimismo, esta estrategia prevé el desarrollo de materiales basados en productos forestales con nuevas propiedades, de forma tal que se dediquen cada vez más bosques a la producción de fibras, madera, energía y a nuevos productos que puedan desarrollarse en el futuro. La estrategia reconoce, no obstante, que incrementos en la extracción de biomasa forestal reducirá la función sumidero de carbono de los bosques, además de afectar potencialmente a una amplia gama de bienes y servicios públicos que los bosques proveen. Por lo que la estrategia bioeconómica forestal europea tiene entre sus objetivos el promover gestiones que incrementen la productividad, el uso eficiente de recursos y la resiliencia de los sistemas forestales, salvaguardando la biodiversidad y evitando comprometer la provisión de los servicios ambientales públicos anteriores (European Commission, 2012: 29).

Una de las políticas específicas que la estrategia bioeconómica europea contempla para garantizar la producción conjunta de productos comerciales, y de bienes y servicios públicos sin mercado, es la de promover una mayor investigación sobre los múltiples servicios de los bosques y su valoración (European Commission, 2012: 31-32). Esta estrategia propone, asimismo, explorar las consecuencias económicas del desarrollo de diferentes sistemas productivos (agrícolas y forestales) en vista de su multifuncionalidad e interacciones intersectoriales, en condiciones climáticas cambiantes.

Por otra parte, la estrategia forestal europea, publicada en 2013, propone un nuevo marco para responder, entre otros retos, a las crecientes demandas de energía y materiales forestales, y a la necesidad de buscar equilibrios con la satisfacción de la demanda de productos tradicionales, y su circunscripción dentro de los límites de la sostenibilidad (Comisión Europea, 2013). Esta estrategia, sienta las bases para hacer compatibles, viables y competitivas la silvicultura con la cadena de valor 
derivada de los bosques, a partir de una serie de objetivos y orientaciones estratégicas. Entre las que se destacan la movilización de fondos y recursos para:

- Estudiar e impulsar otros usos de la madera y productos forestales.

- Transferir nuevos productos y conocimientos científicos y tecnológicos al mercado a fin de dotar de mayor valor añadido a los productos forestales.

- Desarrollar nuevos mercados (dentro y fuera de Europa) para materias primas y productos forestales europeos.

- Mejorar y armonizar los sistemas de información sobre el papel multifuncional de los bosques y de los recursos forestales en Europea.

- Desarrollar e implementar un marco conceptual para la valoración de los servicios ecosistémicos (ambientales), y su integración en los sistemas de contabilidad nacionales y regionales.

Estas recomendaciones destacan la importancia de la investigación en el desarrollo de nuevos productos y mercados para el sector forestal, y la necesidad de ampliar los sistemas estadísticos y contables actuales para evaluar su sostenibilidad. Tanto la estrategia bioeconomica como forestal europeas subrayan la necesidad de producir e integrar indicadores (económicos y/o biofísicos) que ayuden a una mejor comprensión de la contribución de multifuncionalidad de los bosques a la actividad económica y al bienestar social, y de las potenciales pérdidas o ganancias de bienestar por el deterioro o mejora de los ecosistemas forestales y sus recursos.

\subsection{Estrategia bioeconómica española para el sector forestal}

La estrategia bioeconómica española, fue publicada en 2016, y considera que las innovaciones dirigidas al sector forestal tienen un papel fundamental en el cumplimiento de sus objetivos para el horizonte 2030 (MEC, 2016). Esta estrategia menciona concretamente la importancia de promover actividades ligadas a la transformación de la madera, el corcho, la resina, la producción de papel, la obtención de bioenergía, o el uso de residuos de la actividad forestal. Para el ámbito forestal la estrategia bioeconómica española contempla, por un lado, promover el uso de biotecnologías para la mejora genética y la combinación de la madera con otros materiales, a fin de incrementar la durabilidad de los productos madereros; y por otro, favorecer la producción de biomasa como materia prima para las industrias bioquímica y bioenergética. En cuanto a la integración de criterios de sustentabilidad en el manejo forestal, la estrategia española indica su interés en promover modelos productivos que contribuyan a la fijación de carbono y la preservación de la biodiversidad (MEC, 2016).

La estrategia bioeconómica europea da un mayor énfasis a la búsqueda de modelos de producción que permitan garantizar la producción conjunta de productos comerciales, y bienes y servicios públicos sin mercado, así como, a su valoración e integración en sistemas contables, como instrumentos de análisis y gestión de la sustentabilidad forestal. En su lugar, la estrategia bioeconómica española propone basar 
la evaluación de la sustentabilidad a partir de indicadores biofísicos como el volumen total de agua dulce consumida (huella hídrica) o las emisiones de carbono equivalente generadas (huella de carbono) en la producción forestal y procesos bio-industriales [basados en productos forestales, entre otros]. Así como el desarrollo de modelos de evaluación de la sostenibilidad apoyados en indicadores objetivos y comparables. La estrategia española menciona la importancia de garantizar la conservación de la biodiversidad, los ecosistemas y el valor paisajístico, pero de momento no propone medidas concretas para su consideración en la evaluación de sustentabilidad de la gestión forestal.

\section{Retos y Oportunidades bioeconómicas para el sector forestal mediterráneo en España}

España sigue dependiendo en gran medida del petróleo y derivados, que suponen un $52.7 \%$ del consumo total de la energía primaria del país, que es muy superior al consumo medio europeo (26\%) (Eurostat, 2016; MIET, 2016) ${ }^{2}$. No obstante, se ha experimentado un incremento significativo en el consumo de energías renovables desde 1990, duplicando en los últimos 10 años la cuota de estas fuentes energéticas (Rodríguez-Pleguezuelo et al., 2014). En la actualidad esta cuota representa un 6.6\% del consumo de energía final en España, de las que casi tres cuartas partes proceden del uso de biomasa ${ }^{3}$. También cabe destacar que España ocupa el séptimo lugar, en Europa, en la producción y consumo de biomasa para la producción energética (Solid Biomass Barometer, 2015).

Se espera que la demanda de bioenergía de origen forestal crezca en los próximos años, impulsada por los objetivos y políticas de energías renovables en Europa (Hetemamäki, 2014; Rodríguez-Pleguezuelo et al., 2014). Esta demanda ha llevado al auge, por ejemplo, en la producción doméstica, importación y consumo de pellets de madera para generar energía (primordialmente para calefacción) en Europa. En esta región se consumen, en la actualidad, unos 18.8 millones de toneladas de pellets, de los un $47 \%$ se suplen a través de la importación de este producto, principalmente procedente de Norteamérica, Rusia, países europeos fuera de la UE y Sudamérica (Solid Biomass Barometer, 2015).

Las todavía bajas tasa de extracción sobre el crecimiento de la biomasa forestal en Europa, y en España en particular, donde esta tasa es inferior al 35\% (MAAMA, 2014), pueden representar una oportunidad para el desarrollo de centros de producción y procesamiento de biomasa forestal para la producción de pellets, o directamente la generación de electricidad. El desarrollo bioenergético en España requiere, en todo caso, la previa implantación de un sistema interconectado de producción y distribución de biomasa (madera y residuos) y productos derivados; cuyo progreso bá-

\footnotetext{
${ }^{1}$ Datos de 2014, consumo final de petróleo y derivados y gas natural.

${ }^{2}$ Biomasa derivada de los cultivos agrícolas, la actividad forestal, la industria alimentaria, la industria de la madera, del papel y textil, los residuos animales y la fracción orgánica de los residuos sólidos urbanos (MEC, 2016).
} 
sicamente dependerá de la viabilidad y competitividad comercial de estos productos. Así mismo, las bajas tasas de productividad maderera en el monte mediterráneo (Ericsson y Nilsson, 2006) podrían limitar el potencial para una industria forestal bioeconómica en la región. Cabría esperar, por tanto, que la transición hacia una bioeconomía en el sector forestal, como en otros sectores, este condicionada a los avances tecnológicos, los rendimientos técnicos y la rentabilidad de los productos bioeconómicos, así como de la disponibilidad de biomasa a precios competitivos (Scarlat et al. 2015).

En los últimos años se ha observado un creciente interés por estimar el potencial bioenergético basado en la movilización de madera y/o residuos forestales. La Tabla 1 muestra algunos ejemplos recientes de estudios que evalúan la viabilidad técnica y/o económica de incrementar el suministro de biomasa forestal para la producción de energía para calefacción o generación eléctrica a escala local, regional e incluso nacional. A efectos comparativos esta tabla ofrece estimaciones sobre la potencial contribución de la nueva oferta bioenergética al consumo final de energía (en 2015), y la demanda eléctrica y la producción de energías renovables en España en este mismo periodo.

Una serie de estudios aplicados a escala local o regional en España indican un potencial para el uso de biomasa y/o residuos forestales para la producción de calor o electricidad que puede ser relevante localmente. Con excepción del estudio de López-Rodríguez et al. (2009), los estudios regionales presentados en la Tabla 1 se enfocan en bosques atlánticos, con un mayor potencial bioenergético (Turrado Fernández et al., 2016). Los dos estudios aplicados a nivel nacional ofrecen estimaciones divergentes sobre el potencial energético basado en residuos forestales. Las estimaciones de Turrado Fernández et al., (2016) indican un potencial total de 134.13 GJ $\mathrm{Km}^{-2}$ (3.2 tep $\mathrm{Km}^{-2}$ ) para cubrir la demanda de energía (para calefacción) de los hogares españoles, que de realizarse supondría un incremento del $30.5 \%$ sobre el actual consumo final de energías renovables. Gómez et al.(2010), estima un potencial técnico menor, aunque en este caso los residuos forestales se destinan a la producción de electricidad, pudiendo potencialmente cubrir un $4 \%$ de la demanda eléctrica en España.

Los resultados anteriores sugieren posibles conflictos en la demanda de residuos forestales para la producción eléctrica y de calor. En principio, cabría esperar que las decisiones de asignación de productos y residuos forestales dependan de la rentabilidad futura, para el propietario de la tierra, de distintos productos y canales de comercialización, y de la flexibilidad de la gestión forestal para adaptarse a la producción de diferentes bienes. Los estudios consultados ofrecen algunas ideas sobre el potencial económico de la boenergía en España. Por ejemplo, los resultados de Turrado Fernández et al., (2016) sugieren que el uso de residuos forestales para satisfacer la demanda de calefacción en los hogares españoles podría ser competitivo en comparación con otras alternativas energéticas. De acuerdo con este estudio, el coste medio de provisión de residuos forestales en España es de $€ 72,72$ por tonelada seca de biomasa $\left(\sim € 4 \mathrm{Gj}^{-1}\right)$, que es inferior al coste medio del suministro de pellets en las plantas europeas. No obstante, este estudio parece considerar única- 
Tabla 1. Estudios de caso sobre el potencial bioenergético forestal en España.

\begin{tabular}{|c|c|c|c|c|c|c|}
\hline \multirow[t]{3}{*}{ Estudio } & \multirow[t]{3}{*}{ Región } & \multirow{3}{*}{$\begin{array}{l}\text { Descripción } \\
\text { de lasituación } \\
\text { o escenario } \\
\text { analizado }\end{array}$} & \multirow[t]{3}{*}{ Observaciones } & \multicolumn{3}{|c|}{ Potencial bioenergético } \\
\hline & & & & \multirow{2}{*}{$\begin{array}{c}\text { Valor } \\
\text { Ktep }^{(1)} / \text { año }\end{array}$} & \multicolumn{2}{|c|}{ Porcentaje $^{(2)}(\%)$} \\
\hline & & & & & ES2015 & $\Delta \mathrm{ER} 2015$ \\
\hline $\begin{array}{l}\text { Gómez } \\
\text { et al. } \\
(2010)\end{array}$ & España & $\begin{array}{l}\text { Uso de residuos fo- } \\
\text { restal para generar } \\
\text { electricidad } \\
\text { (proceso de estima- } \\
\text { ción jerárquica inte- } \\
\text { grado en un SIG). }\end{array}$ & $\begin{array}{l}\text { Residuos forestales estimados a } \\
\text { partir del IFN para } 18 \text { familias de } \\
\text { especies, considerando diferentes } \\
\text { productividades de residuos por } \\
\text { familia de especies, y restriccio- } \\
\text { nes y límites geográficos (áreas } \\
\text { excluidas) y técnicos (eficiencia } \\
\text { energética) y económicos (costes } \\
\text { de producción de energía). }\end{array}$ & $\begin{array}{l}945 \text { Ktep } \\
\text { año-1(3) }\end{array}$ & 1.08 & 17.84 \\
\hline $\begin{array}{l}\text { López } \\
\text {-Rodríguez } \\
\text { et al. } \\
\text { (2009) }\end{array}$ & Cáceres & $\begin{array}{l}\text { Uso de residuos fo- } \\
\text { restales en una planta } \\
\text { térmica. }\end{array}$ & $\begin{array}{l}\text { Considera el crecimiento de la } \\
\text { biomasa en ramas }<7 \mathrm{~cm} \text { : en } \\
\text { áreas con menos } 20 \% \text { de pen- } \\
\text { diente y a } 3 \mathrm{~km} \text { de caminos o } \\
\text { pistas. }\end{array}$ & 139.00 & 0.16 & 2.62 \\
\hline $\begin{array}{l}\text { Panichelli } \\
\text { y } \\
\text { Gnansounou } \\
(2008)\end{array}$ & $\begin{array}{l}\text { Navarra, } \\
\text { Vizcaya, } \\
\text { Guipúzcoa, } \\
\text { Álava, } \\
\text { La Rioja, } \\
\text { Cantabria } \\
\text { y Burgos }\end{array}$ & $\begin{array}{l}\text { Uso de residuos fo- } \\
\text { restales para producir } \\
\text { madera torrefacta pa- } \\
\text { ra la producción de } \\
\text { electricidad } \\
\text { (métodos basados en } \\
\text { sistemas de informa- } \\
\text { ción geográfica (SIG) }\end{array}$ & $\begin{array}{l}\text { Residuos estimados a partir de } \\
\text { datos del IFN2 e IFN3. Objetivo } \\
\text { es identificar la mejor } \\
\text { localización de } 2 \text { plantas de pro- } \\
\text { ducción energética (las minimi- } \\
\text { zan los costes de producción pre- } \\
\text { cio de la biomasa en finca y cos- } \\
\text { tes de transporte) a las que su- } \\
\text { plen los residuos forestales pro- } \\
\text { ducidos en las provincias estudia- } \\
\text { das }\end{array}$ & $42.13^{(4)}$ & 0.05 & 0.79 \\
\hline $\begin{array}{l}\text { Paredes } \\
\text {-Sánchez } \\
\text { et al. } \\
(2016)\end{array}$ & $\begin{array}{l}\text { Asturias, } \\
\text { (Cuenca } \\
\text { Hullera } \\
\text { Central) }\end{array}$ & $\begin{array}{l}\text { Uso de biomasa y re- } \\
\text { siduos forestales en } \\
\text { un Centro Logístico } \\
\text { de Biomasa (CLB) }\end{array}$ & $\begin{array}{l}\text { Estudio de la viabilidad técnico- } \\
\text { económica del uso de biomasa } \\
\text { forestal }\end{array}$ & 33.16 & 0.04 & 0.63 \\
\hline $\begin{array}{l}\text { Turrado } \\
\text { Fernández } \\
\text { et al. } \\
\text { (2016) }\end{array}$ & España & $\begin{array}{l}\text { La biomasa forestal } \\
\text { disponible es la bio- } \\
\text { masa restante } \\
\text { Después de introducir } \\
\text { restricciones ambien- } \\
\text { tales, económicas y } \\
\text { técnicas a la biomasa } \\
\text { forestal potencial }\end{array}$ & $\begin{array}{l}\text { Biomasa disponible definiendo } \\
\text { nudos de colección local estraté- } \\
\text { gicos por provincia en función de } \\
\text { la productividad de biomasa y } \\
\text { contenido energético, restriccio- } \\
\text { nes ambientales y costes de trans- } \\
\text { porte. }\end{array}$ & $1,615.14^{(5)}$ & 1.84 & 30.50 \\
\hline
\end{tabular}

(1) Miles de tonelada equivalente de petróleo (Ktep) equivalente a 41.868 GJ. (2) Peso del potencial sobre el consumo de energía final en España (ES2015), e incremento sobre la producción de energías renovables en 2015 (LER2015) (MIET, 2016). (3) Potencial técnico de $39.6 \mathrm{PJ}$ al año. (4) Estimación propia considerando 21 GJ por tonelada de biomasa torrefacta (peso seco) y que la demanda total de las dos plantas de torrefacción es de $84 \mathrm{Kt}$ de biomasa (peso seco). (5) Estimado considerando un potencial energético medio de 134.13 GJ Km² (Turrado Fernández et al., 2016: 215) en España, y su superficie (504.782 Km²). 
mente los costes de cosecha, transporte y distribución de biomasa, ignorando aparentemente los costes de transformación de la biomasa en pellets y, en particular, las inversiones necesarias para adoptar los sistemas de calefacción al uso de este tipo de materiales en los hogares españoles. Por su parte, Gómez et al. (2010), apunta a un potencial económico significativamente menor en el uso de residuos forestales para la producción de electricidad, dado que los costes de producción del potencial técnico estimado prácticamente doblan los costes de producción eléctrica en España.

Otro elemento relevante, desde el punto de vista social, para la evaluación del uso de biomasa y/o residuos forestales como alternativa energética es la generación de empleo. Rodríguez-Pleguezuelo et al. (2014), por ejemplo estima que el uso de biomasa para la producción energética ha generado unos 25.355 puestos de trabajo directos e indirectos (datos de 2010), que supondría unos 5 puestos de trabajo cada mil toneladas equivalente de petróleo (Ktep) de bioenergía consumida en España. Este ratio es inferior al generado por las energías eólica y solar fotovoltaica (unos 13 puestos/Ktep y 12 puestos/Ktep, respectivamente), pero superior a las restantes fuentes de energía renovable que se utilizan en España. Considerando el potencial bioenergético estimado por Turrado Fernández et al., (2016), se esperaria que su realizacion genere hasta 8.000 puestos de trabajo. No obstante, esta es una estimación muy general, ya el valor de 5 puestos de trabajo por Ktep considera todas las formas de biomasa, por lo que tendría interés analizar de forma específica la generación de empleo directo en el sector forestal y en los procesos basados en productos y residuos forestales para la producción de energía.

En España el mercado de biomasa es un mercado todavía incipiente, que en la actualidad depende principalmente de pellets de madera y olivos, con distancias transporte considerables que encarecen sus costes producción (Rodríguez-Pleguezuelo et al., 2014; Sayadi et al. 2014). Se espera que en el corto a medio plazo, estas empresas desarrollen estrategias de distribución que garanticen el suministro de pellets a precios competitivos. Alternativas de producción como los cultivos leñosos de corta rotación con especies forestales de crecimiento rápido parecen prometedoras (Rodríguez-Pleguezuelo et al., 2014). No obstante, estas pueden conllevar a un incremento relevante en la demanda de riego, suponiendo una presión importante sobre los recursos hídricos, cuya provisión en el mundo mediterráneo está caracterizada por problemas de escasez e irregularidad (Sayadi et al., 2014). Estos cultivos podrían a la vez competir con la producción de alimentos por los suelos más fértiles, e incluso incrementar el uso de fertilizantes y la contaminación de suelos y aguas.

Son todavía muchos los retos a los que se enfrenta la implantación de mercados bioenergéticos basados en productos y residuos forestales en España. Esta nueva industria se caracteriza, principalmente, por la falta de rentabilidad económica, problemas logísticos y de abastecimiento y por un escaso desarrollo de mercados bioenergéticos (Puy et al., 2008; Sayadi et al., 2014). En algunas regiones, como en Cataluña [y en el norte de España] se añade el problema de una alta fragmentación de las propiedades forestales, que podría dificultar la expansión del uso bioenergético de los 
montes (Puy et al., 2008). La implantación de nuevos sistemas de producción en respuesta a las demandas de bioenergía podría verse también afectada por las preferencias de los propietarios por continuar con sus sistemas de producción actuales (Blennow et al., 2014).

Tradicionalmente la madera se ha utilizado como material de construcción, la fabricación de muebles, como combustible y de forma procesada para la producción de pulpa y papel. La biomasa forestal tiene, no obstante, una multiplicidad de usos y aplicaciones, tales como materiales aislantes, biopolímeros, plásticos y compuestos biológicos, fibras de carbono, productos químicos y textiles basados en celulosa, materiales de embalaje, etc. Los recientes avances, por ejemplo, en la física de materiales, la biología celular y la genómica han permitido nuevas tecnologías de mejora genética de los árboles para mejorar las cualidades de la madera y la resistencia a plagas, o el uso de enzimas para la fabricación de papel. No obstante, muchas de estas tecnologías se encuentran en fase exploratoria y no se han implantado comercialmente. Es posible que en los próximos años, el desarrollo de estos avances técnicos genere nuevas oportunidades para diversificar y revitalizar los mercados de productos forestales, aunque todavía supone muchos grados de incertidumbre en todos los niveles de la cadena de producción.

\section{El papel de los servicios ambientales en una bioeconomía forestal mediterráneo}

En adición a un bajo rendimiento maderero, en muchos montes mediterráneos, la producción de servicios ambientales, como son la protección de cuencas, la conservación de la biodiversidad y valores paisajísticos, la fijación de carbono o la provisión de servicios recreativos, genera un mayor valor social que su explotación comercial (Campos, et al., 2008a; Campos et al. 2008b; Campos et al., 2014; Ovando et al. 2016). Por tanto, no es sorprendente que la producción de madera no sea la actividad productiva principal de una parte relevantes de los bosques mediterráneos de Europa, que se centran más bien en productos forestales no madereros o agroforestales (Nabuurs et al., 2015), y, de forma no necesariamente intencionada, en la provisión de servicios ambientales sin mercado que son disfrutados tanto por sus propietarios, como por sus visitantes y la sociedad en general. Los montes mediterráneos, por otro lado, son uno de los puntos calientes de biodiversidad a escala global (Myers et al., 2000).

Pero ¿qué puede pasar con estos servicios ambientales si en el futuro miles de hectáreas de monte mediterráneo se utilizan para producir monocultivos forestales para generar la producción, por ejemplo, de bioenergía? Existe un amplia literatura científica sobre el impacto de la intensidad y atributos de gestión forestal sobre la biodiversidad (e.g. Biber et al., 2015), la fijación de carbono (e.g. Sedjo y Sohngen, 2012) o la calidad y cantidad de agua (e.g. Vose et al., 2011). Se requiere, sin embargo, mayor investigación para comprender el signo y magnitud de los efectos de incrementos en la movilización de biomasa y residuos forestales y de una intensificación 
en la gestión forestal, sobre la conservación de la biodiversidad, las funciones ecológicas de los ecosistemas forestales, la fertilidad de los suelos o la provisión de recursos hídricos, entre otros. Un mejor conocimiento de estos impactos ambientales es esencial a fin de poder mitigar los problemas que pueden derivarse de una adopción masiva de nuevas estrategias de gestión forestal para la producción de energía y madera.

Algunos de los servicios ambientales que generan los bosques afectan positivamente a la producción de bienes y servicios de otros sectores de la economía sin que medie pago o compensación directa por parte de los sectores y/o agentes beneficiarios. Algunos ejemplos de estos servicios son la regulación de la calidad hídrica, que puede reducir los costes de provisión de agua potable (e.g. Abildtrup et al., 2013), el control de sedimentos que podría afectar positivamente los beneficios de empresas hidroeléctricas (e.g. Arias, et al., 2011), o la conservación de paisaje y biodiversidad podrían suponer oportunidades para el desarrollo ecoturístico. Cambios en la gestión forestal, por tanto, podrían potencialmente afectar la producción de otras actividades económicas. En presencia de conflictos, se pueden (y hacen con cierta frecuencia) establecer pagos por una serie de servicios ambientales a fin de promover formas de gestión compatibles con la provisión de servicios ambientales como los anteriores.

Los pagos por servicios ambientales (PES por sus siglas en inglés) han atraído la atención como mecanismos para traducir las externalidades ambientales fuera del mercado, en incentivos financieros reales para la producción de servicios ambientales (Engel et al. 2008). Desde luego valorar, diseñar y establecer mecanismos de pago o compensaciones por servicios ambientales es una tarea compleja, cuyo funcionamiento y éxito depende de muchos factores, como una correcta identificación de las relaciones causales entre la gestión o protección forestal y la provisión de un servicio ambiental determinado, una clara definición de los derechos de propiedad y uso, una correcta definición de los mecanismos de pago y las obligaciones contractuales o transparencia institucional, entre otros (Engel et al., 2008; Jack et al., 2008). En todo caso, son un ejemplo de la importancia de ampliar la valoración económica de los bosques a otros bienes o servicios más allá de los tradicionales, como así lo reconocen las estrategias forestal y bioeconómica europeas (European Commission, 2012; Comisión Europea, 2013).

El asociar un valor económico al consumo actual, o a las pérdidas o ganancias de bienestar por el deterioro o mejora de los ecosistemas, sus recursos y los servicios que de estos fluyen, es una forma de reconocer explícitamente valores que trascienden los beneficios comerciales privados, y que por diversos fallos y limitaciones de los mercados, como instrumentos de gestión de los recursos, no se ven reflejados en las cuentas, y en su defecto en las decisiones de los individuos, las empresas y las naciones (Turner et al. 1998). Su monetización, con todas las incertidumbres y limitaciones que esta pueda tener, revela los beneficios o costes "ocultos" que la gestión de ecosistemas forestales tiene para la sociedad (e.g. Campos y Caparrós, 2015). La valoración basada en el análisis de las preferencias individuales y sociales, puede generar oportunidades para desarrollar nuevos mercados (en caso de ser viable); así como guiar las políticas e incentivos para una mejor distribución de los recursos pú- 
blicos a la hora de diseñar y desarrollar sistemas de pagos o compensaciones por servicios ambientales que son valorados por la sociedad. La creación de nuevos mercados, y/o pagos por servicios ambientales, hoy fuera del mercado, pueden convertirse en una fuente relevante de ingresos, a la vez que se conservan hábitats, especies y los servicios que fluyen de estos. Por tanto, es deseable ampliar en el futuro la información física y económica relacionada a la producción de bienes y servicios públicos generados por los bosques, y al potencial efecto que nuevas formas de gestión forestal pueden tener sobre los mismos; considerando de forma explícita la incertidumbre asociada a estas estimaciones. Esta información permitirá en el futuro contrastar los beneficios sociales de diferentes opciones para la implantación de modelos forestales que respondan a las nuevas demandas de energía y materiales, y a la demanda de servicios ambientales por parte de la sociedad.

\section{Algunas reflexiones finales}

Algunos estudios sugieren que la movilización de madera y residuos forestales puede suponer una opción competitiva para la producción de pellets y su uso en hogares para calentar agua y calefacción, y en menor medida la generación eléctrica en España. No obstante, la competitividad de estas alternativas energéticas dependerá de:

- Los rendimientos técnicos, y la rentabilidad de la producción, recolección y procesamiento de estos productos para el propietario del monte y las empresas productoras de bioenergía.

- El desarrollo de sistemas interconectados de producción y distribución de biomasa (madera y residuos).

- De las normativas que regulen las actividades forestales en el futuro, incluidas las ambientales, y de la implantación de las políticas energéticas y climáticas europeas, entre otros. Se ignora el potencial técnico y/o económico de nuevas alternativas de gestión forestal para la producción de materiales mejorados para la construcción o las industrias textil y química. Muchos de los avances en los campos de la física de materiales, la biología celular y la genómica para mejorar los rendimientos forestales, la durabilidad de materiales o la resistencia a plagas encuentran o bien en fase exploratoria o al inicio de su implantación comercial en algunas regiones europeas. La todavía falta de experiencias empresariales en la producción y comercialización de bioenergía y nuevos productos forestales, suponen muchos grados de incertidumbre a todos los niveles de la cadena productiva, y una limitada comprensión de los riesgos del desarrollo bioeconómico en el sector forestal.

Destinar mayores recursos públicos y privados a la investigación, desarrollo y mejora de los sistemas de producción, nuevos productos y mercados para el sector forestal es clave para la implantación de un nuevo modelo bioeconómico. Es así mismo fundamental, destinar recursos suficientes para analizar los potenciales riesgos ambien- 
tales y sociales del desarrollo bioeconómico. El desarrollo de un marco conceptual y de un sistema estadístico para la recogida y análisis de indicadores (económicos y/o biofísicos) que permitan mejorar nuestra comprensión sobre la multifuncionalidad de los bosques, y las potenciales pérdidas o ganancias de bienestar por el deterioro o mejora de los ecosistemas forestales y sus recursos se antoja también como un elemento clave para la implantación de un modelo bioeconómico forestal. Se espera que una mayor investigación proporcione las herramientas necesarias a los responsables políticos y otros actores para apoyar la implantación y seguimiento de las estrategias y políticas bioeconómicas a fin de prevenir o mitigar efectos perversos de las nuevas demandas y presiones sobre el sector forestal.

Los problemas de escasez e irregularidad en la provisión de recursos hídricos es una de las mayores limitantes a la mejora de los rendimientos de la madera en los montes mediterráneos. Esta situación puede verse magnificada por el cambio climático, que se espera intensifique las sequias estacionales. La incidencia de incendios forestales es también un factor relevante a considerar a la hora de evaluar diferentes alternativas de producción forestal, en la medida que el riesgo de pérdidas catastróficas puede ser elevado. Ambas condiciones demandarían, en principio, de la selección de especies forestales con mayor resistencia a sequias prolongadas e incendios, aunque estas no necesariamente pudieran las más productivas. Sería deseable, en este sentido, destinar recursos a mejorar los rendimientos productivos de las especies más adaptadas a las condiciones mediterráneas.

Otro de los retos relevantes de la implantación de nuevos modelos de producción forestal es hacerlos compatibles con la provisión de servicios ambientales públicos hoy fuera del mercado, y que son relevantes para el bienestar social. La estimación de las huellas hídricas o de carbono, como propone la estrategia bioeconomica española para evaluar los impactos ambientales de nuevos modelos de gestión forestal, es relevante pero no suficiente a la hora de evaluar otras alternativas económicas para la diversificación productiva del sector forestal. Por tanto sería deseable, como recomienda, en cambio, la estrategia bioeconomica europea, una mayor investigación sobre los múltiples servicios de los bosques y promover su valoración. La valoración basada en el análisis de las preferencias individuales y sociales puede generar oportunidades para desarrollar nuevos mercados o justificar incentivos públicos para la provisión de servicios ambientales. Desarrollar sistemas de información biofísica y económica asociadas a la producción de diversos servicios ambientales en los montes españoles, puede asistir a una mejor planificación territorial en vista de múltiples objetivos y promover una mayor diversificación del sector forestal.

\section{Agradecimientos}

A los organizadores del $7^{\circ}$ Congreso Forestal Español, por su invitación a presentar esta ponencia, y permitirme reflexionar sobre los retos futuros a los que se enfrenta el sector forestal y la sustentabilidad de su gestión. 


\section{Bibliografía}

Abildtrup, J., Garcia, S., \& Stenger, A. (2013). The effect of forest land use on the cost of drinking water supply: A spatial econometric analysis. Ecological Economics, 92, 126-136. http://doi.org/10.1016/j.ecolecon.2013.01.004

Arias, M.E., Cochrane, T.A., Lawrence, K.S., Killeen, T.J., \& Farrell, T.A. (2011). Paying the forest for electricity: a modelling framework to market forest conservation as payment for ecosystem services benefiting hydropower generation. Environmental Conservation, 38(4), 1-12. http://doi.org/10.1017/S0376892911000464

Biber, P., Borges, J., Moshammer, R., Barreiro, S., Botequim, B., Brodrechtová, Y., Sallnäs, O. (2015). How Sensitive Are Ecosystem Services in European Forest Landscapes to Silvicultural Treatment? Forests, 6(5), 1666-1695. http://doi.org/10.3390/f6051666

Blennow, K., Persson, E., Lindner, M., Faias, S.P., \& Hanewinkel, M. (2014). Forest owner motivations and attitudes towards supplying biomass for energy in Europe. Biomass and Bioenergy, 67, 223-230. http://doi.org/10.1016/j.biombioe.2014.05.002

Campos, P., \& Caparrós, A. (2015). Valoración de los servicios públicos y la renta social de los sistemas forestales de Andalucía. Memorias científicas de RECAMAN. Volumen 5. Madrid: Editorial CSIC.

Campos, P., Caparros, A., Begueria, S., Carranza, J., DIaz-Balteiro, L., Diaz, M., Torres, J. (2014). RECAMAN: Manufactured and environmental total incomes of Andalusian forest. Retrieved from http://www.recaman.es/sites/default/files/RECAMAN_SUMMARY_ 02_04_14.pdf

Campos, P., Caparros, A., Oviedo, J.L., \& Ovando, P. (2008). The enviromental value of the forests | La renta ambiental de los bosques. Arbor, (729), 57-69.

Caparros, A., Ovando, P., Oviedo, J. L., \& Campos, P. (2010). Accounting for carbon in avoided degradation and reforestation programmes in Mediterranean forests. Environment and Development Economics, 16(4), 405-428. http://doi.org/10.1017/S1355770X10000082

Comisión Europea. (2010). Libro verde sobre protección de los bosques e información forestal en la UE: Preparación de los bosques al cambio climático.

Comisión Europea. (2013). Una nueva estrategia de la UE en favor de los bosques y del sector forestal. Comunicación de la Comisión al Parlamento Europeo, el Consejo, el Comité Económico y Social Europeo y el Comité de las Regiones.

Comisión Europea. (2014). Un marco estratégico en materia de clima y energía para el periodo 2020-2030. Comunicación de la Comisión al Parlamento Europeo, al Consejo, al Comité Económico y Social Europeo y al Comité de las Regiones. Brusselas. COM(2014) 15 final.

Engel, S., Pagiola, S., \& Wunder, S. (2008). Designing payments for environmental services in theory and practice: An overview of the issues. Ecological Economics, 65(4), 663-674. http://doi.org/10.1016/j.ecolecon.2008.03.011

Ericsson, K., \& Nilsson, L.J. (2006). Assessment of the potential biomass supply in Europe using a resource-focused approach. Biomass and Bioenergy, 30(1), 1-15. http://doi.org/ 10.1016/j.biombioe.2005.09.001

Eriksson, L.O., Löfgren, S., \& Öhman, K. (2011). Implications for forest management of the EU Water Framework Directive's stream water quality requirements - A modeling approach. Forest Policy and Economics, 13(4), 284-291. http://doi.org/10.1016/j.forpol.2011.02.002 
European Commission. (2012). Innovating for Sustainable Growth: A Bioeconomy for Europe. Communication. http://doi.org/10.1017/CBO9781107415324.004

Eurostat. (2016). Energy balance sheets 2014. Publications Office of the European Union (Vol. 33). http://doi.org/10.2785/52802

Eurostat. (2017). Primary production of renewable energy by type. Retrieved June 20, 2017, from http://ec.europa.eu/eurostat/web/products-datasets/-/ten00081

Framstad, E., Berglund, H., Gundersen, V., Heikkilaä, R., Lankinen, N., Peltola, T., Weih, M. (2009). Increased Biomass Harvesting for Bioenergy: Effects on Biodiversity, Landscape Amenities and Cultural Heritage Values. Copenhagen: TemaNord. Nordic Council of Ministers. Retrieved from http://books.google.com/books?hl=en\&lr=\&id=qFHTPxRS43IC\&oi $=$ fnd $\&$ pg $=$ PA $7 \&$ dq $=$ Increased+biomass + harvesting + for+bioenergy+-+effects + on + biodiversity, + landscape + amenities + and + cultural + heritage + values\&ots=D91wn9QcIv\&sig=qz YCdV7_1wZyMld919FOOcHn70I\%5Cnhttp://books

FTP. (2013). Strategic Research and Innovation Agenda for 2020.

García-Ruiz, J.M., López-Moreno, J.I., Vicente-Serrano, S.M., Lasanta-Martínez, T., \& Beguería, S. (2011). Mediterranean water resources in a global change scenario. Earth-Science Reviews, 105(3-4), 121-139. http://doi.org/10.1016/j.earscirev.2011.01.006

Gómez, A., Rodrigues, M., Montañés, C., Dopazo, C., \& Fueyo, N. (2010). The potential for electricity generation from crop and forestry residues in Spain. Biomass and Bioenergy, 34(5), 703-719. http://doi.org/10.1016/j.biombioe.2010.01.013

Häyrinen, L., Mattila, O., Berghäll, S., Närhi, M., \& Toppinen, A. (2017). Exploring the future use of forests: perceptions from non-industrial private forest owners in Finland. Scandinavian Journal of Forest Research, 32(4), 327-337. http://doi.org/10.1080/02827581.2016. 1227472

Häyrinen, L., Närhi, M., Berghäll, S., Mattila, O., \& Toppinen, A. (2013). Exploring the future use of forests in Finland_: perspectives from sustainability oriented forest owners. Manuscript, 0(0), 1-15. http://doi.org/10.1080/02827581.2016.1227472

Hetemäki., L. (2014). Introduction. In Hetemäki. L. (Ed.), What Science Can Tell Us. Future of the European Forest-Based Sector: Structural Changes Towards Bioeconomy (pp. 11-14). European Forest Institute.

Hetemäki, L., Hoen, H.F., \& Schwarzbauer, P. (2014). Future of the European Forest- Based Sector and Bioeconomy. ThinkForest Brief. Retrieved from http://www.efi.int/portal/virtual_library/publications/what_sci- ence_can_tell_us/6/

Hetemäki, L., \& Hurmekoski, E. (2014). Forest Products market outlook. In L. Hetemäki (Ed.), What Science Can Tell Us. Future of the European Forest-Based Sector: Structural Changes Towards Bioeconomy (pp. 15-32). European Forest Institute.

Hetemamäki, L. (ed. (2014). Future of the European Forest-Based Sector: Structural Changes Towards Bioeconomy. What Science Can Tell Us. Future of the European Forest-Based Sector: Structural Changes Towards Bioeconomy.

Jack, B.K., Kousky, C., \& Sims, K.R.E. (2008). Designing payments for ecosystem services: Lessons from previous experience with incentive-based mechanisms. Proceedings of the National Academy of Sciences, 105(28), 9465-9470. http://doi.org/10.1073/pnas.070550 3104

Kellomäki, S., Kilpeläinen, A., \& Alam, A. (2013). Forest Bioenergy Production. Managament, Carbon sequestration and Adaptation. (S. Kellomäki, A. Kilpeläinen, \& A. Alam, Eds.). London: Springer. 
López-Rodríguez, F., Atanet, C.P., Blázquez, F.C., \& Celma, A.R. (2009). Spatial assessment of the bioenergy potential of forest residues in the western province of Spain, Caceres. Biomass and Bioenergy, 33(10), 1358-1366. http://doi.org/10.1016/j.biombioe.2009.05. 026

MAAMA. (2014). Diagnóstico del sector forestal Español. Análisis Y Porspectiva, 8(Octubre), $1-10$.

Mayumi, K. (2001). The Origins of Ecological Economics: The Bioeconomics of GeorgescuRoegen. Ecological Economics. http://doi.org/10.1016/S0921-8009(02)00129-5

MCPFE, UNECE, \& FAO. (2007). State of Europe's forests 2007. The MCPFE report on sustainable forest management in Europe. Warsaw: Ministerial Conference on the Protection of Forests in Europe, United Nations Economic Commission for Europe, ood and Agriculture Organization of the United Nations. http://doi.org/10.1017/CBO9781107415324.004

Ministerio de Economía y Competitividad (MEC). (2016). Estrategia Española De Bioeconomía: Horizonte 2030. Retrieved from bioeconomia.agripa.org

Ministerio de Industria Energía y Turismo (MIET). (2016). La Energía en España 2015.

Mola-Yudego, B., Arevalo, J., Díaz-Yáñez, O., Dimitriou, I., Freshwater, E., Haapala, A., Selkimäki, M. (2017). Reviewing wood biomass potentials for energy in Europe: the role of forests and fast growing plantations. Biofuels, 7269(March), 1-10. http://doi.org/ 10.1080/17597269.2016.1271627

Myers, N., Mittermeier, R.A., Mittermeier, C.G., Da Fonseca, G.A.B., \& Kent, J. (2000). Biodiversity Hotspots for Conservation Priorities. Nature, 403(6772), 853-858.

Olesen, A. S., Kittler, B., Price, W., \& Aguilar, F. X. (2016). Environmental Implications of Increased Reliance of the EU on Biomass from the South East US. http://doi.org/10.2779/ 30897

Ovando, P., Campos, P., Oviedo, J.L., \& Caparrós, A. (2016). Ecosystem accounting for measuring total income in private and public agroforestry farms. Forest Policy and Economics, 71, 43-51. http://doi.org/10.1016/j.forpol.2016.06.031

Palahi, M., Mavsar, R., Gracia, C., \& Birot, Y. (2008). Mediterranean forests under focus. International Forestry Review, 10(4), 676-688. http://doi.org/10.1505/ifor.10.4.676

Panichelli, L., \& Gnansounou, E. (2008). GIS-based approach for defining bioenergy facilities location: A case study in Northern Spain based on marginal delivery costs and resources competition between facilities. Biomass and Bioenergy, 32(4), 289-300. http://doi.org/ 10.1016/j.biombioe.2007.10.008

Paredes-Sánchez, J.P., García-Elcoro, V.E., Rosillo-Calle, F., \& Xiberta-Bernat, J. (2016). Assessment of forest bioenergy potential in a coal-producing area in Asturias (Spain) and recommendations for setting up a Biomass Logistic Centre (BLC). Applied Energy, 171, 133-141. http://doi.org/10.1016/j.apenergy.2016.03.009

Pedroli, B., Elbersen, B., Frederiksen, P., Grandin, U., Heikkilä, R., Krogh, P.H., Spijker, J. (2013). Is energy cropping in Europe compatible with biodiversity? - Opportunities and threats to biodiversity from land-based production of biomass for bioenergy purposes. Biomass and Bioenergy, 55, 73-86. http://doi.org/10.1016/j.biombioe.2012.09.054

Rodríguez-Pleguezuelo, C.R., Zuazo, V.H.D., Bielders, C., Bocanegra, J.A.J., PereaTorres, F., \& Martínez, J.R.F. (2014). Bioenergy farming using woody crops. A review. Agronomy for Sustainable Development, 35(1), 95-119. http://doi.org/10.1007/s13593-014-0262-1

San Miguel-Ayanz, A. (2005). Mediterranean European silvopastoral systems. Silvopasotralism and Sustainable Management. Lugo, NW Spain.- April 2004, (April), 1-6. 
Sayadi-Gmada, S., Durán-Zuazo, V.H., \& Parra-López, C. (2014). ¿Es posible la producción de biomasa de cultivos leñosos de rotación corta en Andalucía? Agricola Vergel, 33(375), 209-212.

Scarlat, N., Dallemand, J.F., Monforti-Ferrario, F., \& Nita, V. (2015). The role of biomass and bioenergy in a future bioeconomy: Policies and facts. Environmental Development, 15, 3-34. http://doi.org/10.1016/j.envdev.2015.03.006

Sedjo, R., \& Sohngen, B. (2012). Carbon Sequestration in Forests and Soils. Annual Review of Resource Economics, 4, 127-153. http://doi.org/10.1079/PAVSNNR20094041

Sikkema, R., Dallemand, J.F., Matos, C.T., van der Velde, M., \& San-Miguel-Ayanz, J. (2016). How can the ambitious goals for the EU's future bioeconomy be supported by sustainable and efficient wood sourcing practices? Scandinavian Journal of Forest Research, 0(0), 1-8. http://doi.org/10.1080/02827581.2016.1240228

Sjostrom, E. (2013). Wood Chemistry: Fundamentals and Applications (Second Edi). Academic Press.

Söderberg, C., \& Eckerberg, K. (2013). Rising policy conflicts in Europe over bioenergy and forestry. Forest Policy and Economics, 33, 112-119. http://doi.org/10.1016/j.forpol.2012. 09.015

Solid Biomass Barometer. (2015). EurObserv'ER. Retrieved from https://www.eurobserver.org/

Turner, R.K., Adger, W.N., \& Brouwer, R. (1998). Ecosystem services value, research needs, and policy relevance: A commentary. Ecological Economics, 25(1), 61-65. http://doi.org/ 10.1016/S0921-8009(98)00018-4

Turrado Fernández, S., Paredes Sánchez, J.P., \& Gutierrez Trashorras, A.J. (2016). Analysis of forest residual biomass potential for bioenergy production in Spain. Clean Technologies and Environmental Policy, 18(1), 209-218. http://doi.org/10.1007/s10098-015-1008-8

Vicente-Serrano, S.M., Lasanta, T., \& Gracia, C. (2010). Aridification determines changes in forest growth in Pinus halepensis forests under semiarid Mediterranean climate conditions. Agricultural and Forest Meteorology, 150(4), 614-628. http://doi.org/10.1016/j.agrformet.2010.02.002

Vose, J.M., Sun, G., Ford, C.R., Bredemeier, M., Otsuki, K., Wei, X., Zhang, L. (2011). Forest ecohydrological research in the 21st century: what are the critical needs? Ecohydrology, 4(2), 146-158. http://doi.org/10.1002/eco.193 